\title{
Urgences
}

\section{Dispositio et dispositifs : l'invention poétique dans La figue (sèche) de Francis Ponge}

\section{Bernard Beugnot}

Numéro 24, juillet 1989

Le manuscrit sous l'angle

URI : https://id.erudit.org/iderudit/025531ar

DOI : https://doi.org/10.7202/025531ar

Aller au sommaire du numéro

Éditeur(s)

Urgences

ISSN

0226-9554 (imprimé)

1927-3924 (numérique)

Découvrir la revue

Citer cet article

Beugnot, B. (1989). Dispositio et dispositifs : l'invention poétique dans La figue (sèche) de Francis Ponge. Urgences, (24), 43-51. https://doi.org/10.7202/025531ar d'utilisation que vous pouvez consulter en ligne.

https://apropos.erudit.org/fr/usagers/politique-dutilisation/ 


\section{Dispositio et dispositifs: l'invention poétique dans La figue (sèche) de Francis Ponge}

\section{Bernard Beugnot, Université de Montréal}

La perspective génétique bénéficie d'une double légitimation dans l'œuvre poétique de Ponge. Par le geste inaugural de publier les dossiers, partiels ou exhaustifs, de plusieurs textes, depuis La rage de l'expression (1952) jusqu'à La table (1981).' Par la thématique ou le mouvement de structuration qui anime les textes les plus amples: Le galet, 1928; Le soleil placé en abime, 1928-1954; La Mounine ou note après coup sur un ciel de Provence, 1941; Le lézard, 1945-1947.2 Situation plus symptômatique que totalement singulière: Louis Hay a déjà noté que les poètes sont à l'origine de la préoccupation génétique. ${ }^{3}$

1 De nombreux manuscrits partiels sont reproduits en fac-similés dans diverses éditions. Pour un inventaire, on se reportera à François Chapon, "Catalogue des manuscrits de F. Pongen, Francis Ponge. Manuscrits. Livres Peintures, 25 février-4 avril 1977, Paris, Centre Beaubourg.

2 L'exploitation des manuscrits connus, dans une perspective génétique, n'a pas encore fait l'objet de travaux nombreux. Sont à noter: 1980 Jean-Marie Gleize et Bernard Veck, F. Ponge. Actes ou textes, Presses de l'Université de Lille (Sur le dossier partiel, La fabrique du pré, Genève, Skira, 1971); 1980 Jean-Pierre Richard, «Fabrique de la figue», Critique, juin-juillet, nos 397-198, p. 551-570 (sur le dossier intérgal publié par Jean Ristat, Comment une figue de paroles et pourquoi, Paris, Flammarion, 1977); 1987 Thomas Aron, «La réécriture faite texte: un moviment de Ponge, La Tablem, Annales littéraires de l'Université de Besançon, p. 179-212 (Sur le dossier intégral de La table, publié par B. Beugnot et R. Mélançon, Études françaises, 17, avril 1981, p. 3-49 et Montréal, Éditions du silence, 1982).Sur la génétique du texte pongien, voir: B. Beugnot, "Pongepaysage: genèse de La Mounine", Saggi e ricerche di letteratura francese (sous presse); Poétique de Ponge. Le palais diaphane, Paris, PUF, «Ecrivains" (sous presse).

3 aLa critique génétique: origines et perspectives", Essais de critique génétique, Paris, Flammarion, p. 227-236. Plusieurs collectifs ont été depuis consacrés à la critique génétique, en particulier: Avanttexte, texte, après-texte, Paris, CNRS, 1982; La genèse du texte. Les 
Dans le large champ de la génétique, mon titre, encore trop général, ouvre un territoire qui appellerait de longues analyses sur le seul dossier de La figue. Je me contenterai d'esquisser une problématique sur quelques exemples ponctuels, en regardant non pas la naissance des formulations, des idées ou des thèmes, mais la réorganisation du matériau textuel rendu disponible par les réécritures premières. Bien que ces deux procès de l'invention poétique ne soient pas, dans la réalité, dissociés de façon absolue, la trame chronologique partage la gestation du texte en deux grands moments.

Après trois notes initiales espacées ${ }^{4}$, cinq campagnes d'écriture ${ }^{5}$ constituent l'invention proprement dite, c'est-à-dire l'accumulation et la mise en forme progressive des matériaux lexicaux, thématiques, stylistiques. Campagne d'écriture désigne ici une suite de feuillets dont les dates montrent qu'il n'y a pas eu d'interruption de travail supérieure à quelques jours. Mais ces campagnes sont de durée très inégale et correspondent à des types différents de travail textuel. Ainsi à compter de septembre 1958 - le 13 une nouvelle rédaction s'intitule «Der des der» et le 20 apparaît la première copie dactylographiée -, Ponge se livre à ce qu'il appelle lui-même des "essais de mise en page". Le 9 novembre, une nouvelle copie dactylographiée se donne comme "dernier état" et, à partir de cette date, il vagabonde avec des "gloses annexes", des textes sur la figue fraîche ou sur la datte dont rien ne passera dans les réécritures ultimes.

Une nouvelle étape s'amorce où commence véritablement le travail de disposition, sans que Ponge s'interdise les

modèles linguistiques, Paris, CNRS, 1982; Leçons d'écriture. Ce que disent les manuscrits. Hommage à Louis Hay, Paris, Minard, 1985; De la genèse du texte littéraire, Paris, Du Lérot, 1988.

4 Ces trois notes et les états du 31 mars au 7 avril 1958 font l'objet d'un recopiage «très corrigé» (J. Ristat) dans un petit carnet, inséré dans le dossier entre les feuillets du 29 août et du 1 er septembre. La démarche fait problème, si le carnet n'a pas été déplacé, car Ponge est un diariste scrupuleux qui note toujours avec soin le moment de l'écriture ou de la réécriture. Est-ce le sentiment provisoire que le texte est mûr et qu'il n'y a plus qu'à mettre en scène le travail jusque-là mené de l'inventio?

5 En 1958, du 31 mars au 10 avril, du 7 au 12 juin, du 8 août au 21 septembre, du 9 novembre au 17 décembre. En 1959, du 8 février au 22 avril. 
reformulations; la dernière campagne d'écriture est suivie de vingt-cinq états dactylographiés, non datés, qui se présentent comme autant de tentatives de "version finale". Le dossier est ainsi constitué, presque pour moitié, de dactylogrammes qui vraisemblablement s'échelonnent tout au long de l'année 1959. La copie remise à Tel Quel et les pages de Tel Quel corrigées en vue de la réédition dans Le grand recueil (1961) ferment le tout.

Avant de regarder de plus près la combinatoire à laquelle se livre Ponge, quelques précisions terminologiques sont indispensables. Par séquence, j'entends une unité textuelle identifiable soit par sa cohérence thématique, soit par sa récurrence comme ensemble répérable en divers lieux du dossier; la séquence correspond souvent à un changement de point de vue ou de registre stylistique. Le 7 septembre 1958, Ponge en dresse une liste qui a pour titre "La figue (strophes de)". Appuyé généralement sur des indications explicites du manuscrit, le découpage des séquences est un premier facteur de variabilité dans la disposition puisque d'un état à l'autre il établit des rapports instables avec les phrases, les paragraphes et la typographie. La notion de séquence, malgré ce qu'elle comporte de conventionnel ou d'arbitraire, est un outil méthodologique commode pour suivre le travail de la réécriture.

La dispositio, traditionnelle troisième partie de la rhétorique classique, vise en termes aristotéliciens "l'économie du discours" ou, selon Quintilien, "la répartition fonctionnelle des choses et des parties en divers lieux". S'y choisit l'ordre de succession des séquences en même temps que s'y poursuit une fin esthétique.

Quant aux dispositifs, il faut entendre le terme en son sens militaire, c'est-à-dire la formation adoptée, ici formation textuelle, en vue d'une stratégie et de la production de divers effets sémantiques ou visuels. À la dispositio qui ordonnance, les dispositifs ajoutent la présentation graphique dans la page ou celle des phrases à l'intérieur de la séquence. L'invention poétique s'exerce donc, une fois accumulées les formulations et les thèmes, sur un parcours, sur des rythmes et sur des espaces.

Seul l'examen de ces hésitations et oscillations, qui représentent autant de solutions possibles du problème textuel que pose la description de la figue, permet d'entrer dans l'atelier et de caractériser l'invention pongienne. 
Les espacements de la version de Tel Quel, déterminés par Ponge, divisent le texte en neuf séquences de longueur très inégale (de une à vingt lignes). Trois seulement suffiront à montrer les effets qui tiennent à la dispositio: la première qui noue la figue à une réflexion sur la poésie; la troisième qui la compare à une chapelle romane; la cinquième, très brève, qui énonce la moralité poétique.

La première réunit des formulations primitives dont elle inverse l'ordre d'émergence. Dès février 1951, la figue est qualifiée de "pauvre gourde", mais son "art poétique" ne devient explicite que le 17 avril. Désormais les deux thèmes vont naviguer de conserve en contraste, ou en contrepoint, le privilège de l'inaugural étant accordé tantôt au fruit, tantôt à son allégorie. Double visage qui d'emblée impose le modèle de la fable à une séquence qui figure parmi les plus stables.

La troisième est celle dont les éléments constitutifs connaissent le plus de variations rédactionnelles et de migrations. La structure allégorique s'y retrouve dans la friction d'un plan descriptif ou comparatif et d'un plan discursif, chacun disposant de sa nappe lexicale propre: thème des tentatives et des approximations, métaphore de la chapelle enfouie. Pauvreté et richesse, misère et éclat, la réunion en cette séquence de formulations venues de diverses versions, exploitant l'opposition "pauvre gourde / autel scintillant" (février 1951), vise à constituer la figue en objet oxymorique. Ainsi installée au cœur du texte, l'imagerie religieuse se diffracte sur tout le texte dont elle représente le noyau métaphorique essentiel. Liée aux images chères à Ponge du temple et du Louvre, instrument indirect de l'éloge de la poésie, elle appelle le langage religieux, encomiastique par nature. Déjà la version élaborée entre juillet et septembre 1958 avait, par sa disposition prosodique, surtout dans la séquence II, apparenté plus étroitement le texte à un hymne ou à une litanie.

La cinquième séquence - «Ainsi de l'élasticité à l'esprit des paroles, - et de la poésie comme je l'entends" - connaît, elle, diverses transhumances entre l'ouverture et la chute du texte (28 août 1958). Le 16 décembre 1958, dans un état en cinq séquences clairement numérotées, elle disparaît, sans doute pour éviter la redondance avec la formule initiale qui déclare l'allégorie: "La figue sèche est le modèle ou l'exemple de nos savoureuses difficultés d'ici bas", mais elle refait surface avec les tentatives de version complète qui commencent en avril 
1959. Si la place ne change pas la portée, elle modifie le statut de cette moralité. Apparue le 11 juin 1958, dans une version courte, comme variante de l'expression "épaisseur élastique" de quelques mois antérieure, elle s'énonçait comme la leçon finale d'une fable laconique, La figue sèche ou la consolation matérialiste. Réinsérée dans le corps du texte, elle sert à en clore une phase dont les séquences suivantes deviennent la paraphrase et servent à convertir la fable en parabole par recours insistant au registre religieux. Autant d'effets de la dispositio: phrases ou fragments de texte, tôt trouvés ou peu retravaillés, cherchent leur place dans des ensembles variables et mobiles.

Quant aux dispositifs, il faudrait pour en juger placer sous les yeux plusieurs états. Quelques constats suffiront. En premier lieu, la version datée du 20-21 septembre 1958 est déjà très proche de celle qui sera confiée à Tel Quel. Le mouvement de l'invention n'est donc ni linéaire, ni continu, fait de régressions, de tentatives insatisfaisantes; les rapports qu'entretiennent l'inspiration - entendons les formulations et l'organisation textuelle venues précocement ou rapidement - et le travail - entendons les efforts systématiques de réécriture - sont tendus et conflictuels. Les essais de dispositifs ne sont pas seulement la poursuite d'un texte idéal ou rêvé, mais l'épreuve de genres nouveaux et de diverses configurations mimétiques. «Poésie aplatie" le 4 novembre 1958: version descriptive très sommaire où la structure allégorique n'est plus qu'allusivement présente pour qui connaît le reste du dossier. Plus tard (p. 179, sd), un état également très bref qui se clôt sur la séquence II, retrouve à la fois le modèle de la fable et celui du Parti pris des choses: tout l'essentiel y est dit dans le dépouillement d'une description qu'encadre l'oxymore "pauvre gourde / autel scintillant".

En bref, les divers dispositifs entre lesquels oscillent les essais de mise en page modifient à chaque fois les rapports du poète à son objet et à son lecteur, mais surtout correspondent à autant d'approximations possibles de la figue que sa nature oxymorique interdit de fixer et à autant de genres qui partiellement l'épousent. D'où l'épaisseur d'un dossier qui finalement jamais ne se clôt. Les oppositions de rythme, contraction / dilution, systole / diastole, le resserrement ou l'étirement des paragraphes, l'aération typographique qui retrouve les chemins de la prosodie qui alterne avec une 
brevitas toute sénéquienne, autant de manières de traduire, en un texte visuel, les tensions ou les exigences contraires de la description et du discours sur la poésie, du rythme de la prose et de celui de la poésie entre lesquels hésite le proème, de se faire image d'un fruit qui est à la fois l'un et le multiple, "pauvre gourde" et "grenier à tracasserie pour les dents".

L'apparence lisse et neutre du poème paru dans Tel Quel, dont le Grand recueil (1961) donnera juste une version resserrée, dissimule ces multiples tensions auxquelles le manuscrit donne accès. L'entrée dans l'atelier confère rétrospectivement à la lecture une densité et une force allusive neuves. Gros de sa genèse, le texte n'est jamais qu'un compromis.

Processus ou procédés, ces démarches de l'invention ne sont pas sans modèles. Bien que Ponge juge le calligramme insuffisant (My creative Method, 1948), il exprime plusieurs fois son admiration pour Apollinaire dont il a lu la conférence au Vieux Colombier en novembre 1917 sur "L'esprit nouveau et les poètes". Les deux états imprimés d'un passage du Savon révèlent sa vive sensibilité à la portée visuelle et sémantique du dispositif typographique. Dans la version publiée par Le disque vert (1953) la scriptio continua déporte l'attention de la langue et du poétique vers le figuratif, contraignant l'esprit à extraire le sens du "buisson typographique" (Les múres, 1935) de la même manière que l'on "extirpe une savonnette de son enveloppe de papier" 6 . L'imaginaire pongien aime jouer de ces analogies. En revanche, l'état de 1967 réfère à l'exemple mallarméen du Coup de dés qui avait été au principe de l'Araignée (1942-1948):

L'avantage de cette distance copiée qui mentalement sépare des groupes de mots ou les mots entre eux, semble d'accélérer tantôt et de ralentir le mouvement, le scandant, l'intimant même selon une vision simultanée de la page. (Préface de 1897)

L'invention poétique de Ponge ne joue pas seulement avec la recherche lexicale ou phrastique, mais aussi avec l'aménagement spatial du texte alors même qu'il se situe en dehors de toute prosodie. Le manuscrit et ses états multiples

6 Le néologisme architexte semble n'être qu'un intensif qui déclare cette multiplicité d'effets de sens. II n'a en tout cas rien à voir avec le terme qu'emploiera G. Genette en 1979. 
donnent accès non seulement au ductus de l'écriture, mais à une physiologie du trait où se troublent ses frontières avec le dessin. ${ }^{7}$

Ainsi l'avènement du texte - encore que l'expression, entachée de téléologie, exprime mal la nature du trvail pongien dont un dossier manuscrit exhaustif donne le spectacle s'opère au carrefour de plusieurs systèmes qu'il est difficile de suivre conjointement, mais que Ponge explore souvent simultanément.

Pour fins de discussion, je retiendrai de ces observations hâtives les points suivants:

- Si l'imprimé, avec ses variations de format, de mise en page, de typographie modifie le visage et la réception d'un texte ${ }^{8}$, le manuscrit de Ponge avec ses multiples essais de mise en page explore de manière plus systématique tous les possibles et modifie le caractère et le statut de la version publiée. Celle-ci est moins un état définitif qu'un suspens provisoire, l'arrêt sur un possible, sans pourtant que cet arrêt soit tout à fait aléatoire puisque Ponge parle de nœud ou de version complète, c'est-àdire celle où sont reprises, au moins de manière allusive, le plus grand nombre de séquences et celle où se nouent la description et le sens allégorique, conjonction qui ouvre en même temps sur la meilleure réalisation esthétique, car la beauté “c'est le rapport de la forme exactement, figurativement, minutieusement décrite, à une vérité d'esprit. C'est le rapport de la forme de chaque objet avec l'art poétique de cet objet." (7 avril 1958). Au terme de variante trop statique et ponctuelle qui renvoie implicitement surtout à une différence de rédaction, ou de variation que Ponge emprunte au registre musical ("L'art de la figue"), je voulais substituer celui de variance ${ }^{9}$, à cause de la valeur du suffixe qui

7 aJe sens ma main agir, tourner, lier, plonger, se lever et bien souvent, par le jeu des corrections, raturer ou faire éclater la ligne, agrandir l'espace jusqu'à la marge, construisant ainsi, à partir de traits menus et apparemment fonctionnels (les lettres) un espace qui est tout simplement de l'art.» (R. Barthes, préface à La civilisation de l'écriture, de F. Druet et H. Grégoire, Paris, Fayard, 1980).

8 Roger Chartier et Christian Jouhaud, «Pratiques historiennes des textes*, L'interprétation des textes, sous la direction de Claude Reichler, Paris, Editions de Minuit, 1989 (en particulier p. 60 et 66).

9 Ce suffixe désigne, en électrodynamique, ale comportement d'un conducteur par rapport au courant $(\mathrm{H}$. Cottez, Dictionnaire des structures du vocabulaire savant, Paris, Le Robert, 1980). 
dit admirablement la modification des rapports entre un invariant - en l'occurrence les séquences - et ses supports, ici les dispositifs. B. Cerquiglini m'a devancé. ${ }^{10}$ /I reste que l'expression d' "élasticité des paroles" dont la figue est l'emblème poétique invite à une rêverie étymologique et sémantique: métaphore, selon l'origine grecque, de la poussée créatrice qu'est la rage de l'expression, aptitude du mot et du texte à "céder à une pression et reprendre sa forme" (Littré), tension manifeste dans tout le dossier entre la «résistance" et la "non résistance», dans cette alternance d'expansion et de resserrement.

- Éditer scientifiquement l'œuvre de Ponge impliquerait que l'on recuse le modèle reçu de l'édition critique au profit d'une double édition: commentée pour repérer les réminiscences, les sources, les allusions, les autocitations extrêmement nombreuses qui constituent en réseau de cette œuvre en apparence disparate; édition génétique à inventer puisque le dossier semble revenir à la variabilité médiévale. ${ }^{11}$

- La transcription imprimée très imparfaite, malgré conventions typographiques et fac-similés, des originaux sur lesquels il faudrait travailler, laisse néanmoins bien voir deux modes de l'invention. L'un récurrent dans la plupart des manuscrits accessibles où reviennent les mêmes attitudes, les mêmes gestes: recours au Littré ou à des encyclopédies pour y puiser des références, des définitions, des citations qui interviennent comme confirmation ou enrichissement en cours de travail plutôt que comme moteur initial; pratique de la réécriture systématique, du recopiage avec des degrés de correction très variables, dont la fonction semble être de réamorçage du travail d'écriture, en particulier au début de chaque campagne.ll y a chez Ponge une habitude la reduplicatio ou de l'anadiplose généralisée.

Le second mode d'invention, plus spécifique, est lié aux pressions et aux exigences de chaque texte singulier, selon la formule désormais bien galvaudée, "une rhétorique par objet".

10 Éloge de la variante. Histoire critique de la philologie, Paris, Seuil, 1989.

11 Voir les pages de conclusion de B. Cerquiglini et B. Beugnot, aLa "rage de l'expression": considérations éditoriales sur les dossiers de F. Ponge», La naissance du texte, colloque international, Paris, CNRS, 1987 (Prétirage, p. 195-200). 
Ainsi le travail de la dispositio et de la disposition n'a été, à ma connaissance, nulle part aussi poussé que dans La figue. Aporie qui tient peut-être à une adéquation trop parfaite à un objet qui enveloppe une multitude de grains d'or et qui est fait pour "être mâchonné sans fin comme on fait des bons textes" (21 septembre 1958).

L'accès à un manuscrit comme celui de La figue (sèche) est sans doute moins important par ce qu'il nous apprend de la chronologie d'une genèse d'où émergerait le "bon texte" que par ce qu'il révèle d'incertitude dans la prolifération de ce qu'il faut appeler versions réservées plutôt qu'avant-textes. Déçus dans la quête d'une origine, nous voici, témoins médusés, devant le tremblement d'une invention poétique pour laquelle faire choix d'une disposition est figer le mouvement et par là régresser ou renoncer à toutes les virtualités de l'expresion. Le vrai texte n'est finalement pas sans doute celui qui a reçu, en revue ou en livre, la sanction de l'impression, mais celui qui, quelque part entre les états et versions multiples, attend une naissance qui ne viendra jamais; texte de nulle part, utopique comme le Livre mallarméen. 